\title{
对话陈声: 关注细菌耐药
}

我国是抗菌药物最大的生产和使用国. 抗菌药物被称 为最伟大的发明之一, 并被广泛应用于医疗卫生、农业养 殖等领域, 在治疗感染性疾病、防治动物疫病、提高养殖 效益和保障公共卫生安全等方面发挥重要作用. 但是近些 年, 细菌耐药事件频频发生, 世界卫生组织多次呼叮合理 使用抗生素. 为使大众了解细菌耐药性的一些相关知识, 《科学通报》对话深圳市食品生物性安全防控研究重点实 验室主任、香港理工大学食物安全及技术研究中心副主任、 香港食物安全中心专家组成员、香港理工大学陈声教授.

陈声，2000 2004年在美国马里兰大学攻读博士学位, 从事食源性病原菌耐药机理的研究; 2004 2009年在美国 威斯康星医学院开展博士后研究工作, 从事细菌致病性和 毒素结构功能研究; 2009年至今在香港理工大学先后任助 理教授、副教授和教授，长期从事微生物病原菌耐药机制、 致病机制及新抗生素开发等研究. 已主持国家重点基础研 究发展计划(973)1项、深圳市重点实验室项目1项, 香港研 究资助局、传染病控制专项基金和香港理工大学研究项目 16项, 在New Engl J Med, Lancet Infect Dis, Nat Commun, Pro Natl Acad Sci USA, Chem Comm等学术刊物上发表论 文97篇, 研究论文被国际期刊他引超过 2560 次, 获美国发 明专利2项。

《科学通报》: 请您简要介绍一下目前国内外细菌 耐药性的研究及发展情况.

陈声：2000年我从中国农业大学硕士毕业后到美国马 里兰大学攻读博士学位, 主要研究方向就是细菌的耐药 性. 我的第一个研究课题是从肉类食品中分离并鉴定携带 $b l a_{\mathrm{CMY}-2}$ 型 $\beta$-内酰胺酶的沙门菌, 该研究结果发表在 2001 年的New Engl J Med ${ }^{[1]}$. 这也说明17年前, 细菌耐药性还 普遍较低, 而且广受人们的重视. 随后的一个课题是比较 从美国和中国不同来源分离到的大肠杆菌和沙门菌的耐 药性差异. 研究发现, 从中国分离到的菌株对氟喹诺酮抗 生素的耐药性比美国分离株要高; 而中国分离到的菌株对 头孢菌素类抗生素耐药性为零, 美国分离的部分菌株对头 孢菌素类抗生素已经开始产生耐药, 这与两个国家当时的 畜牧业抗生素使用情况吻合. 在中国, 氟哇诺酮类抗生素 已经广泛应用于食品动物的养殖中, 而头孢菌素类抗生素 由于价格偏高, 很少在动物上使用. 与之相反, 美国对氟 喹诺酮类药物在动物上的使用控制较为严格, 然而头狍类

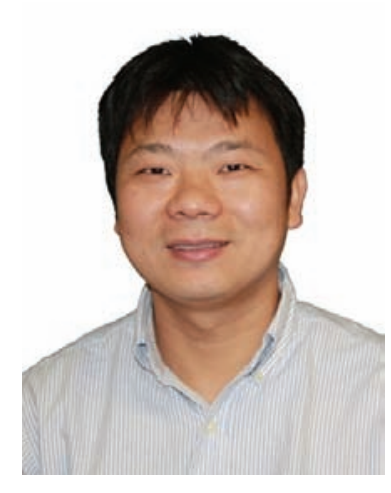

香港理工大学教授陈声

抗生素头孢噻呋当时已被批准用于动物疾病治疗. 抗生素 使用的不同导致细菌耐药谱在两个国家存在巨大差别, 同 时从侧面说明了在动物中使用氟喹诺酮类和头孢菌素类 抗生素是推动细菌耐药性产生和流行的主要原因. 西方国 家对病原菌的耐药性一直以来都非常重视，美国于1996年 成立了国家耐药性监测系统(NARMS), 对重要食源性病原 菌的耐药性进行长期监测. 监测数据表明这些重要食源性 病原菌的耐药性总体维持在较低水平, 例如沙门菌对头孢 类抗生素的耐药性在 $4 \%$ 左右, 对氟喹诺酮的耐药低于 $1 \%$. 然而, 近 10 年是中国细菌耐药性快速发展的阶段, 细菌对 临床重要抗生素的耐药率呈直线上升的趋势. 令人担忧的 是, 目前这种上升趋势并未缓解, 反而促使耐药菌向着高 毒力和高传播的方向发展, 给养殖业、环境和人体健康构 成巨大的威胁.

《科学通报》: 细菌耐药性相对比较宽泛, 不同的细 菌与不同的药物间会有不同的耐药性. 请您介绍一下 目前对大众影响较大的抗生素及其耐药菌间的初步研 究进展.

陈声: 氟喹诺酮和头孢类抗生素是较早使用的抗菌药 物. 在肠杆菌科细菌中, 目前造成头孢类抗生素耐药最为 流行的是CTX-M型超广谱 $\beta$-内酰胺酶(ESBL). CTX-M型超 广谱 $\beta$-内酰胺酶于 2002 年首次在我国临床大肠杆菌中报 道 ${ }^{[2]}$, 并于 2006在香港和内地的动物大肠杆菌中分离. 随 后几年, CTX-M型超广谱 $\beta$-内酰胺酶在动物和临床大肠杆 菌中快速传播. 我们近两年对动物源大肠杆菌的研究发 现, 鸡肉和猪肉中分离的大肠杆菌的 $b l a_{\mathrm{CTX}-\mathrm{M}}$ 基因阳性率 
接近 70\%, 而养殖场猪和鸡粪便中分离大肠杆菌的 $b l a_{\mathrm{CTX}-\mathrm{M}}$ 阳性率超过 95\%. 对于临床分离菌, 2016年的 CHINET数据表明其大肠杆菌ESBL检出率高达 $45 \%$.

沙门菌是人和动物重要的病原菌, 而氟喹诺酮是用于 治疗临床沙门菌感染的重要药物. 氟喹诺酮耐药的沙门菌 分别于 2008 和 2010 年首次在我国临床和动物的沙门氏菌 中报道; 头狍噻呋耐药的沙门菌在2009和2010年分别在临 床和动物的沙门菌中报道. 以往的研究显示沙门氏菌的氟 喹诺酮耐药主要是由于 $g r y A$ 基因的双重突变导致. 然而沙 门氏菌 $g r y A$ 基因的双重突变很少发生, 因此沙门氏菌氟喹 诺酮耐药很低. 而我们最近的研究发现临床分离鼠伤寒沙 门菌对氟喹诺酮耐药率从 2006年的 $20 \%$ 上升到2013年的 45\%. 究其原因, 主要为质粒介导的喹诺酮耐药基因的产 生. 沙门氏菌可通过携带多种质粒介导的喹诺酮耐药基因 来替代 $g r y A$ 基因的双重突变而产生氟喹诺酮耐药. 这一新 的耐药机制的出现, 使得沙门菌的治疗少了一种重要的抗 生素选择 ${ }^{[3,4]}$.

霍乱弧菌、副溶血弧菌等是引起腹泻重要的病原菌. 过去几年其对常用抗生素尤其是对氟喹诺酮和头狍类抗 生素的耐药性一直保持较低水平. 但近年来耐药性也逐渐 上升, 我们实验室从2011年开始持续跟踪弧菌的耐药性变 化, 到2016年, 弧菌氟喹诺酮的耐药率已达到 30\%左右, 其中靶基因突变和携带不同类型的 $q n r V C$ 基因是介导氟喹 诺酮耐药的主要机制. 弧菌对头狍类的耐药率在2016年达 到 $20 \%$ 左右. 有意思的是, 介导头孢类抗生素耐药的机制 在不断的变化, 从最早发现的PER-1头孢菌素酶到CMY-2 到 VEB-2 又到了在肠杆菌科细菌中最流行的 CTX-M类 $\mathrm{ESBL}^{[5 \sim 7]}$. 在此过程中前面的头狍菌素酶渐渐消失, 新出 现的不断增加. 介导这种耐药进化的机制还是一个谜, 有 待进一步研究.

碳青霉烯类抗生素是治疗ESBL等耐药菌极为有效的 药物, 然而其耐药率也快速上升. 根据2016 CHINET耐药 监测网数据, 我国临床肺炎克雷伯菌的碳青電烯耐药率从 2005 年的 $3 \%$ 上升到 2016 年的 $18.6 \%$. 而碳青霉烯酶NDM-1 和KPC-2 的传播是导致碳青霉烯耐药率上升的主要因素. 对耐药机制的进一步研究表明, 携带KPC- 2 的质粒具有多 样性, 而携带NDM-1基因的质粒主要是由IncX3型质粒介 导的. 而不管相对多样性的 KPC- 2 介导质粒或相对单一性 的NDM- 1 介导质粒, 其携带 KPC- 2 或NDM- 1 基因的移动元 件是非常保守的, 说明了这些保守移动元件的转移是介导 碳青霉烯耐药性传播的主要因素 ${ }^{[8]}$. 由于碳青霉烯类抗生 素耐药率的不断上升, 临床上也出现了高毒力、高耐药和 高传播力的肺炎克雷伯菌. 这些病原菌人血后可造成高死 亡率, 而这类菌的治疗成为临床最为棘手的问题.
另外一个非常重要的药物为多黏菌素, 多黏菌素被认 为是治疗碳青需烯耐药菌的最后一道防线. 事实上多黏菌 素是一剂“老药”, 在我国耳肾毒性多黏菌素仅仅在动物上 使用, 在临床上尚未开始使用. 近期我国会逐渐停止在农 业上使用多黏菌素, 而开始应用于临床抗多重耐药菌的治 疗. 但是由于长期在动物上使用,多黏菌素的耐药率不断 增加. 我国科学家 2015年底发现了耐多黏菌素的 $m c r-1$ 基 因. 自此, 它已在世界各地 30 多个国家被报道. $m c r$ - 1 基因 多数情况下由可接合的质粒介导, 并在大肠杆菌和其他细 菌中广泛传播. 目前研究表明, $m c r-1$ 主要由 3 种不同类型 的质粒介导. 然而携带 $m c r-1$ 基因的移动元件也非常保守, 而且可以形成环状中间体，并介导 $m c r-1$ 基因在不同质粒 甚至染色体上转移 ${ }^{[9,10]}$. 虽然 $m c r-1$ 基因对多黏菌素的疗效 的影响尚未明确, 但其传播严重威胁了多黏菌素作为细菌 感染的最后一道防线的地位.

除了革兰阴性菌, 革兰阳性菌的耐药性问题也非常值 得关注. 金黃色葡萄球菌是医院感染重要的病原菌, 当金 黄色葡萄球菌以神奇的速度变成耐甲氧西林金黃色葡萄 球菌(MRSA), 随后耐万古霉素的MRSA陆续被报道, 让全 世界预感超级细菌年代的来临. 值得庆幸的是, 耐万古霉 素金黃色葡萄球菌仍然很低, 可能金黃色葡萄球菌很难产 生万古霉素耐药. 最近科学家们又发现了几种可治疗革兰 阳性菌的新抗生素, 似乎金黄色葡萄球菌的治疗不是难 题. 但是, 肠球菌的耐药悄然出现, 其对万古需素的耐药 逐年上升, 对新药达托需素的耐药也快速上升. 另外一个 潜在的问题是: 造成肠球菌万古需素耐药的机制是否将来 有一天会在金黃色葡萄球菌适应并传播, 这将会是另一个 世界性抗感染治疗难题.

《科学通报》: 据了解, 细菌耐药性仍在高速发展, 基因技术也逐步运用到细菌耐药性研究, 能否为我们 简单介绍相关的研究进展?

陈声: 研究细菌耐药性近 20年以来, 我深切感受到细 菌耐药的严重性. 而近10年我国细菌耐药性到了一个高速 发展的时期, 从临床最常用的氟喹诺酮和头孢类抗生素到 抗感染最后防线的碳青霉烯类抗生素和多黏菌素, 细菌都 可以从自然界中找到最好的应对方法, 就是各种各样的耐 药基因. 这些耐药基因都是源于不同的细菌, 是细菌用于 竞争和自身生存的遗传因素, 并属于这些特定的细菌. 当 细菌在长期抗生素的选择压力下, 这些耐药基因的两端被 装上不同的转座酶基因, 形成可移动的元件. 同时细菌染 色体外遗传物质如噬菌体和质粒也在长期抗生素的选择 压力下进行进化和重组, 形成不同种类的可接合质粒. 携 带耐药基因的移动元件可被整合到不同的质粒上, 并在不 
同种属的细菌中进行转移, 极大地加速细菌耐药性的产生 和发展. 随着过去几十年的积累, 这种携带耐药基因的移 动元件的种类越来越多, 同时耐药质粒的种类也越来越 多. 当自然界中这种类似“积木”的材料的种类越来越多时, 细菌就可以组装出超级质粒以介导多种抗生素的耐药性. 借助于现代二代和三代测序技术的进步, 我们得以获得各 种各样的质粒序列来研究多重耐药质粒的组成和进化. 结 果几乎令我们绝望. 细菌可在一种质粒上装上各种各样的 携带不同耐药基因的移动元件, 产生一个介导多重耐药的 超级质粒. 一个敏感细菌在获得这个质粒后可马上变成一 个超级细菌. 更为严重的是, 不同转座酶还可介导质粒重 组, 将两种或多种不同质粒整合成一个质粒, 使它变成一 个可适应多种宿主的广谱耐药质粒. 随着质粒和耐药移动 元件的种类增加, 细菌可在各种各样的生态环境和小生境 中, 如水和土壤环境、动物和人的肠道环境、医院环境和 食物环境等进行质粒组合和进化, 快速组装出适应不同种 属细菌的多重耐药的可接合质粒. 今后细菌耐药性的发展
会变得越来越快和越来越难以控制.

《科学通报》: 最后, 请结合您的研究数据预测一下 未来细菌耐药性的发展状况.

陈声: 我个人认为细菌耐药性的问题才刚刚开始, 在 不久的将来会变得更为严重. 人类回到前抗生素时代或进 人后抗生素时代的预言将很快实现或已经到来. 随着因细 菌感染而死亡的患者的增加，临床上不得不使用更好的抗 生素，在没有更有效的后备药物的情况下，人类医学将退 回原始年代, 一个简单的手术将可使人致命. 人和病原菌 的战争是长期的, 寻找解决细菌耐药性的方法不仅仅是全 世界科学家的最紧要任务, 也是全世界人们要共同面对的 问题. 目前全世界各个国家、世界卫生组织等机构已经开始 尝试不同的计划, 如减少动物使用抗生素、停止在动物上使 用人用重要抗生素如多黏菌素、临床上合理使用抗生素等措 施. 期望通过这些措施来缓解细菌耐药性的问题, 并不断 探索和研究, 希望有一天能够找到行之有效的解决方法.

(张冬梅 王元火)

\section{推葆阅读文献}

1 White D G, Zhao S, Sudler R, et al. The isolation of antibiotic-resistant salmonella from retail ground meats. New Engl J Med, 2001, 345: $1147-1154$

2 Chanawong A, M'Zali F H, Heritage J, et al. Three cefotaximases, CTX-M-9, CTX-M-13, and CTX-M-14, among Enterobacteriaceae in the People's Republic of China. Antimicrob Agents Chemother, 2002, 46: 630-637

3 Lin D, Chen K, Wai-Chi Chan E, et al. Increasing prevalence of ciprofloxacin-resistant food-borne Salmonella strains harboring multiple PMQR elements but not target gene mutations. Sci Rep, 2015, 5: 14754

4 Wong M H, Chan E W, Liu L Z, et al. PMQR genes oqxAB and aac (6') Ib-cr accelerate the development of fluoroquinolone resistance in Salmonella typhimurium. Front Microbiol, 2014, 5: 521

5 Li R, Ye L, Zheng Z, et al. Genetic characterization of a blaVEB-2-carrying plasmid in Vibrio parahaemolyticus. Antimicrob Agents Chemother, 2016, 60: 6965-6968

6 Li R, Lin D, Chen K, et al. First detection of AmpC beta-lactamase bla (CMY-2) on a conjugative IncA/C plasmid in a Vibrio parahaemolyticus isolate of food origin. Antimicrob Agents Chemother, 2015, 59: 4106-4111

7 Li R, Wong M H, Zhou Y, et al. Complete nucleotide sequence of a conjugative plasmid carrying bla (PER-1). Antimicrob Agents Chemother, 2015, 59: 3582-3584

8 Zhang R, Liu L, Zhou H, et al. Nationwide surveillance of clinical carbapenem-resistant enterobacteriaceae (CRE) strains in China EBioMedicine, 2017, 19: 98-106

9 Li R, Xie M, Zhang J, et al. Genetic characterization of mcr-1-bearing plasmids to depict molecular mechanisms underlying dissemination of the colistin resistance determinant. J Antimicrob Chemother, 2017, 72: 393-401

10 Li R, Xie M, Lv J, et al. Complete genetic analysis of plasmids carrying mcr-1 and other resistance genes in an Escherichia coli isolate of animal origin. J Antimicrob Chemother, 2017, 72: 696-699 